\title{
Expression of receptor for advanced glycation end products (RAGE) on the surface of circulating endothelial cells is upregulated in Kawasaki disease
}

\author{
Fangqi Gong' ${ }^{1}$ Ye Zhang ${ }^{1}$, Chunhong Xie' ${ }^{1}$, Weihua Zhu' ', Wei Wang ${ }^{1}$, Songling Fu' and Hongqiang Shen ${ }^{2}$
}

INTRODUCTION: The aim of this study was to investigate the expression of receptor for advanced glycation end products (RAGE) on the surface of circulating endothelial cells (CECS) in patients with Kawasaki disease (KD).

METHODS: The positive rate of RAGE on the surface of CECS (CECs-RAGE/CECS) and the fluorescence intensity of RAGE on the surface of CECs (FI-RAGE-CECS) were evaluated in 89 patients with $K D$ in the acute stage (A-KD), subacute stage (SA-KD), or convalescent stage (C-KD).

RESULTS: CECs-RAGE/CECs and the FI-RAGE-CECs increased significantly in patients with KD. The CECs-RAGE/CECs was significantly higher in C-KD patients with coronary artery lesions (CALs) than in those without CALs. The FI-RAGE-CECs level was significantly higher in SA-KD and C-KD patients with CALs than in A-KD patients. In SA-KD and C-KD patients, the CECs-RAGE/ CECs and FI-RAGE-CECs levels decreased in intravenous immunoglobulin (IVIG)-respondent patients but increased progressively in IVIG-resistant patients and were significantly higher in IVIG-resistant patients than in IVIG-respondent patients.

DISCUSSION: The results suggest that the expression levels of RAGE on the surface of CECs are upregulated in KD patients, and that the upregulated expression levels of RAGE on the surface of CEC s can be aggravated in SA-KD and C-KD patients with CALS, and also in IVIG-resistant SA-KD and C-KD patients. The RAGE expression on CECs is involved in the pathophysiology of KD.

K awasaki disease (KD), an acute febrile disease that usually occurs in children under 5 y of age (1), has become the primary cause of acquired heart disease in children $(2,3)$. KD affects predominantly small- and medium-size arteries, particularly the coronary artery (4), with potential risks for coronary stenosis and thrombosis caused by coronary artery lesions (CALs), and even death caused by myocardial infarction $(5,6)$. CALs can be found in about $10-15 \%$ of children with KD (7). Although the treatment with intravenous immunoglobulin (IVIG) for patients with $\mathrm{KD}$ in the acute stage (A-KD) can reduce the duration of fever and the incidence of CALs, 10\% of children with $\mathrm{KD}$ do not respond to IVIG treatment and therefore have higher risk for CALs $(8,9)$. Both the etiology and pathogenesis of KD remain unclear (10).

Endothelial damage is the basic pathologic process in the development of many diseases. Circulating endothelial cells (CECs) represent a new sensitive indicator for studying vascular endothelial function in vivo (11). CALs caused by KD are closely related to the function of coronary artery endothelial cells, and the CEC levels increase significantly in the blood of patients with A-KD (12).

S100A12 is mostly expressed in human granulocytes. S100A12 exerts proinflammatory effects by eliciting a transient infiltration of neutrophils and more delayed recruitment of monocytes at inflammatory sites, activating endothelial cells and leukocytes, and potently chemoattracting monocytes, which is a key process, especially in vasculitis (13-19). S100A12 is the first member of the $S 100$ protein family shown to engage the multiligand receptor for advanced glycation end products (RAGE) and to exert its regulatory effects on target cells via RAGE binding. RAGE engagement by S100A12 in leukocytes and endothelial cells results in activation of nuclear factor $-\kappa B$ with resulting activation of proinflammatory genes. S100A12/RAGE interactions appear to be functionally important in human pathologies (20-22). Many studies showed that S100A12 has a close relation with KD (23-26). Our studies found that CECs increased significantly in KD patients; the S100A12 expression on the surface of CECs increased significantly in KD patients and persisted for a longer time in patients with CALs (27), raising the possibility that S100A12 might have a role in the pathophysiology of KD via RAGE engagement.

There has been no report on the expression of RAGE on the surface of CECs in patients with KD. To determine whether there is relationship between KD and RAGE on the surface of CECs, we investigated the positive rate of RAGE on the surface of CECs (CECs-RAGE/CECs), and the fluorescence intensity (FI) of RAGE on the surface of CECs (FI-RAGE-CECs) by monoclonal antibody combined with flow cytometry in KD patients with or without CALs, and in IVIG-respondent or IVIG-resistant KD patients in acute (A-KD), subacute (SA-KD), and convalescent (C-KD) stages. 


\section{RESULTS}

\section{CECs-RAGE/CECs and FI-RAGE-CECs Levels in Patients With KD}

The CECs-RAGE/CECs and FI-RAGE-CECs levels were significantly $(P<0.05)$ higher in KD patients than in the controls and did not decrease significantly in SA-KD and C-KD patients (Table 1).

\section{CECs-RAGE/CECs and FI-RAGE-CECs Levels in KD With CALs}

The CECs-RAGE/CECs and FI-RAGE-CECs levels were significantly $(P<0.05)$ higher in KD patients with CALs than in the controls. The FI-RAGE-CECs levels were significantly $(P<0.05)$ higher in SA-KD and C-KD patients than in $\mathrm{A}-\mathrm{KD}$ patients (Table 2).

\section{CECs-RAGE/CECs and FI-RAGE-CECs Levels in IVIG-Resistant KD} The CECs-RAGE/CECs and FI-RAGE-CECs levels were significantly $(P<0.05)$ higher in IVIG-resistant KD patients than in the controls. The CECs-RAGE/CECs were significantly $(P$ $<0.05)$ higher in C-KD patients than in A-KD and SA-KD patients $(P<0.05)$. The FI-RAGE-CECs levels increased progressively in SA-KD and C-KD patients (Table 3 ).

\section{Comparison of CECs-RAGE/CECs and FI-RAGE-CECs Levels in KD} With and Without CALs

The CECs-RAGE/CECs rate was significantly $(P<0.05)$ higher in $\mathrm{C}-\mathrm{KD}$ patients with CALs than in those without CALs. The FI-RAGE-CECs levels were significantly $(P<0.05)$ lower in A-KD patients with CALs than in those without CALs and

Table 1. CECs-RAGE/CECs and FI-RAGE-CECs in patients with KD

\begin{tabular}{llcl}
\hline & $n$ & CECs-RAGE/CECs (\%) & Fl-RAGE-CECs \\
\hline A-KD & 89 & $26.70 \pm 10.02^{\mathrm{a}}$ & $31.20 \pm 11.43^{\mathrm{a}}$ \\
SA-KD & 89 & $23.76 \pm 6.38^{\mathrm{a}}$ & $29.95 \pm 8.92^{\mathrm{a}}$ \\
C-KD & 89 & $25.05 \pm 9.85^{\mathrm{a}}$ & $28.91 \pm 8.43^{\mathrm{a}}$ \\
Control & 38 & $17.71 \pm 4.52$ & $20.86 \pm 4.16$ \\
\hline
\end{tabular}

A-KD, Kawasaki disease in the acute stage; C-KD, Kawasaki disease in the convalescent stage; CECs-RAGE/CECs, the positive rate of RAGE on the surface of circulating endothelial cells; FI-RAGE-CECs, the fluorescence intensity of RAGE on the surface of circulating endothelial cells; RAGE, receptor for advanced glycation end products; SA-KD, Kawasaki disease in the subacute stage.

a $P<0.05$ as compared with control.

Table 2. CECs-RAGE/CECs and FI-RAGE-CECs in KD patients with CALs

\begin{tabular}{lccl}
\hline & $n$ & CECs-RAGE/CECs (\%) & FI-RAGE-CECs \\
\hline A-KD & 15 & $25.68 \pm 7.41^{\mathrm{a}}$ & $25.91 \pm 8.20^{\mathrm{a}}$ \\
SA-KD & 15 & $25.67 \pm 7.29^{\mathrm{a}}$ & $30.51 \pm 12.98^{\mathrm{a}, \mathrm{b}}$ \\
C-KD & 15 & $27.92 \pm 13.03^{\mathrm{a}}$ & $30.14 \pm 8.64^{\mathrm{a}, \mathrm{b}}$ \\
Control & 38 & $17.71 \pm 4.52$ & $20.86 \pm 4.16$ \\
\hline
\end{tabular}

A-KD, Kawasaki disease in the acute stage; C-KD, Kawasaki disease in the convalescent stage; CALs, coronary artery lesions; CECs-RAGE/CECs, the positive rate of RAGE on the surface of circulating endothelial cells; FI-RAGE-CECs, the fluorescence intensity of RAGE on the surface of circulating endothelial cells; RAGE, receptor for advanced glycation end products; SA-KD, Kawasaki disease in the subacute stage.

${ }^{a} P<0.05$ as compared with control; ${ }^{b} P<0.05$ as compared with $A-K D$. decreased progressively in SA-KD and C-KD patients without CALs (Figure 1).

\section{Comparison of CECs-RAGE/CECs and FI-RAGE-CECs Levels in IVIG-Respondent and IVIG-Resistant KD Patients}

In SA-KD and C-KD, the CECs-RAGE/CECs and FI-RAGECECs levels were significantly $(P<0.05)$ higher in IVIGresistant patients than in IVIG-respondent patients. But the FI-RAGE-CECs level was significantly $(P<0.05)$ lower in IVIG-resistant A-KD patients than in IVIG-respondent A-KD patients. The CECs-RAGE/CECs and FI-RAGE-CECs levels decreased in IVIG-respondent SA-KD and C-KD patients but increased progressively in IVIG-resistant SA-KD and C-KD patients (Figure 2).

\section{DISCUSSION}

In this study, we found that the expression levels of RAGE on the surface of CECs in patients with KD were upregulated and did not decrease significantly in SA-KD or C-KD; the expression levels of RAGE were similar to the expression levels of S100A12 found on the surface of CECs in patients with KD (27). This suggests that the high levels of S100A12 on the surface of CECs may stimulate the expression of RAGE on the surface of CECs in patients with KD. This study corroborates the concept that the expression of RAGE is upregulated in presence of its own ligand by a positive feedback loop mechanism, that RAGE participates in the pathophysiology of KD, and that the S100A12-RAGE pathway may be crucial in the pathogenesis of KD.

Several studies (14) demonstrate that the activation of RAGE by S100A12 results in upregulated expression of proinflammatory cytokines, such as tumor necrosis factor and interleukin-1 $\beta$, by other phagocytes. Released tumor necrosis factor may stimulate granulocytes to secrete S100A12, thereby establishing a self-amplifying positive feedback loop. S100A12activated peripheral blood mononuclear cells release interleukin-2 and show enhanced expression of cytokines (14). RAGE expression increases whenever its ligands accumulate (20), and RAGE-ligand interaction leads to physiological and pathological processes, including diabetic complications, neurodegenerative disorders, atherosclerosis, and inflammation $(21,28)$. However, the full extent of RAGE expression and the molecular

Table 3. CECs-RAGE/CECs and FI-RAGE-CECs in IVIG-resistant KD

\begin{tabular}{lccl}
\hline & $n$ & CECs-RAGE/CECs $(\%)$ & Fl-RAGE-CECs \\
\hline A-KD & 7 & $24.42 \pm 12.88^{\mathrm{a}}$ & $26.58 \pm 7.76^{\mathrm{a}}$ \\
SA-KD & 7 & $26.50 \pm 6.81^{\mathrm{a}}$ & $33.41 \pm 13.85^{\mathrm{a}, \mathrm{b}}$ \\
C-KD & 7 & $31.97 \pm 15.32^{\mathrm{a}, \mathrm{c}}$ & $38.67 \pm 12.34^{\mathrm{a}, \mathrm{b}, \mathrm{d}}$ \\
Control & 38 & $17.71 \pm 4.52$ & $20.86 \pm 4.16$
\end{tabular}

A-KD, Kawasaki disease in the acute stage; C-KD, Kawasaki disease in the convalescent stage; CECs-RAGE/CECs, the positive rate of RAGE on the surface of circulating endothelial cells; FI-RAGE-CECs, the fluorescence intensity of RAGE on the surface of circulating endothelial cells; IVIG, intravenous immunoglobulin; RAGE, receptor for advanced glycation end products; SA-KD, Kawasaki disease in the subacute stage.

a $P<0.05$ as compared with control; ${ }^{b} P<0.05$ as compared with $A-K D ; c P<0.05$ as compared with A-KD and SA-KD; ${ }^{\mathrm{d} P}<0.05$ as compared with SA-KD. 

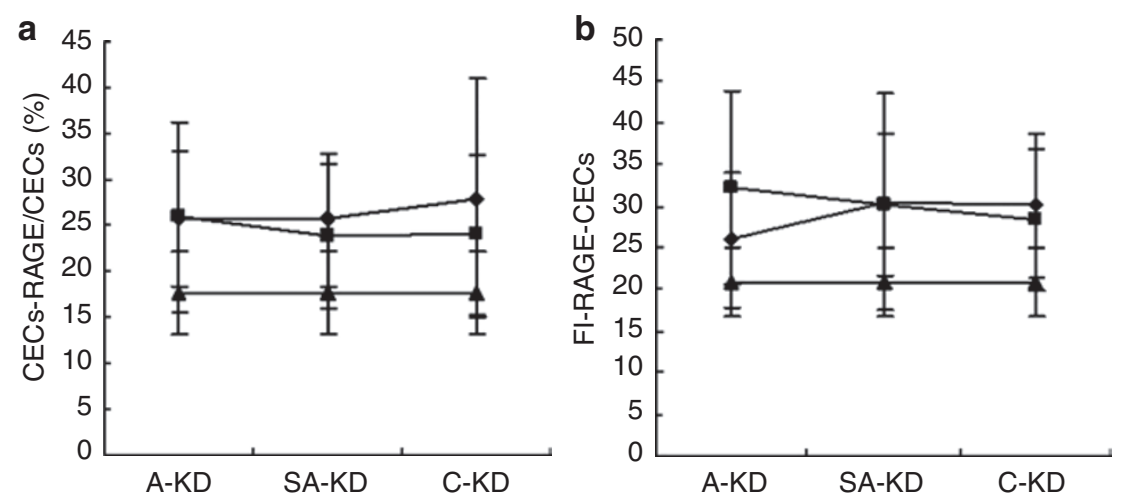

Figure 1. Diagram of CECs-RAGE/CECs and FI-RAGE-CECs levels in KD patients with or without CALs. (a) CECs-RAGE/CECs levels; (b) FI-RAGE-CECs levels. CECs-RAGE/CECs, the positive rate of RAGE on the surface of circulating endothelial cells; FI-RAGE-CECs, the fluorescence intensity of RAGE on the surface of circulating endothelial cells; A-KD, Kawasaki disease in the acute stage; SA-KD, Kawasaki disease in the subacute stage; C-KD, Kawasaki disease in the convalescent stage. Shaded diamonds, KD with CALs; shaded squares, KD without CALs; shaded triangles, controls. CALs, coronary artery lesions; CECs, circulating endothelial cells; FI, fluorescence intensity; KD, Kawasaki disease; RAGE, receptor for advanced glycation end products.
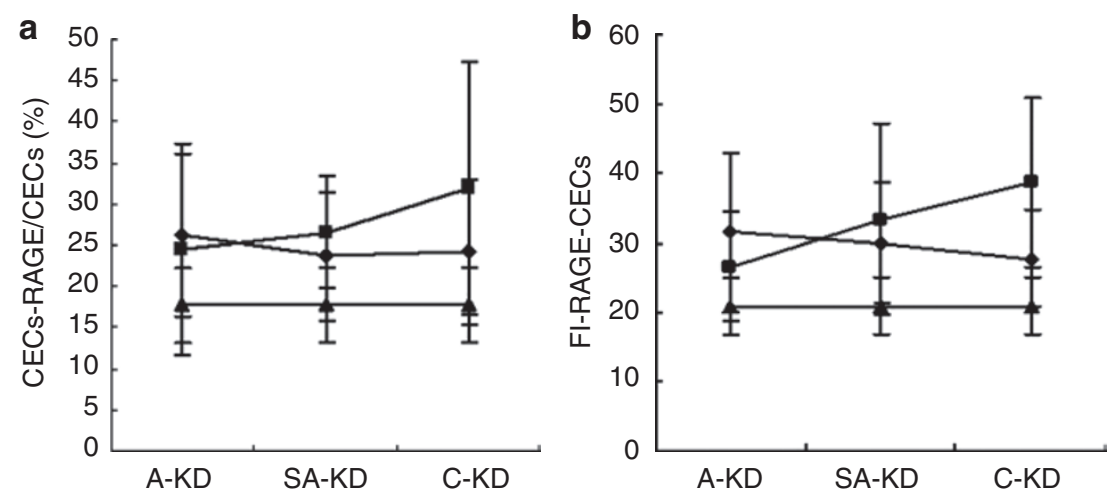

Figure 2. Diagram of CECs-RAGE/CECs and FI-RAGE-CECs levels in IVIG-respondent and IVIG-resistant KD patients. (a) CECs-RAGE/CECs levels; (b) FI-RAGE-CECs levels. CECs-RAGE/CECs, the positive rate of RAGE on the surface of circulating endothelial cells; FI-RAGE-CECs, the fluorescence intensity of RAGE on surface of circulating endothelial cells; A-KD, Kawasaki disease in the acute stage; SA-KD, Kawasaki disease in the subacute stage; C-KD, Kawasaki disease in the convalescent stage. Shaded diamonds, IVIG-respondent; shaded squares, IVIG-resistant; shaded triangles, controls. CALs, coronary artery lesions; CECs, circulating endothelial cells; Fl, fluorescence intensity; IVIG, intravenous immunoglobulin; KD, Kawasaki disease; RAGE, receptor for advanced glycation end products.

mechanisms that control it have not been evaluated adequately. Understanding the role of RAGE in development could provide insights to pursue the reduction of RAGE-mediated CALs in KD.

Mahajan et al. reported that C-reactive protein upregulated the expression of RAGE in a dose- and time-dependent manner in the THP-1 monocytic cell line (29). Zhong et al. reported that the expression of RAGE in human endothelial cells was found to be increased in the presence of $\mathrm{C}$-reactive protein and suggested that C-reactive protein enhances the ability of RAGE to bind to its ligands (30). The clinical characteristics of this group of KD patients showed that C-reactive protein increased significantly in A-KD and decreased significantly in SA-KD. We did not find the correlation between C-reactive protein levels and the expression levels of RAGE on the surface of CECs in patients with $\mathrm{KD}$. However, it is possible that $\mathrm{C}$-reactive protein can stimulate the expression of RAGE on the surface of CECs in A-KD patients. Although C-reactive protein levels decrease quickly in SA-KD patients, the elevated expression levels of
RAGE on the surface of CECs in patients with KD can persist for a longer time.

The study found that the FI-RAGE-CECs levels were higher in SA-KD and C-KD patients than in A-KD patients with CALs, but decreased progressively in SA-KD and C-KD patients without CALs; the CECs-RAGE/CECs rate was higher in C-KD patients with CALs than in those without CALs. This suggests that the endothelial cell lesions can be alleviated in SA-KD and C-KD patients without CALs and can be aggravated in SA-KD and C-KD patients with CALs. It is possible that the persistence of inflammation is longer in KD patients with CALs than in those without CALs. The FI-RAGE-CECs levels were significantly lower in IVIG-resistant A-KD patients than in IVIG-respondent A-KD patients. In IVIG-resistant SA-KD and C-KD patients, the CECs-RAGE/CECs and FI-RAGE-CECs levels increased progressively and were higher than in IVIG-respondent SA-KD and $\mathrm{C}-\mathrm{KD}$ patients. This illustrates that the expression levels of RAGE on the surface of CECs in patients with KD is correlated with IVIG-resistant or IVIG-respondent patients, and 
the effect by which IVIG reduces the pathological changes of endothelial cells is weaker in IVIG-resistant patients than in IVIG-respondent patients. This suggests that the inflammation is more severe in KD patients with CALs and IVIG-resistant KD patients than in KD patients without CALs or IVIG-respondent KD patients. It demonstrates further that the S100A12-RAGE pathway participates in the pathogenesis of $\mathrm{KD}$.

In summary, the expression levels of RAGE on the surface of CECs are upregulated in KD patients. The upregulated expression levels of RAGE on the surface of CECs can be alleviated in SA-KD and C-KD patients without CALs and can be aggravated in SA-KD and C-KD patients with CALs. In IVIGresistant SA-KD and C-KD patients, the expression levels of RAGE on the surface of CECs increase progressively and are higher than in IVIG-respondent SA-KD and C-KD patients. This suggests that RAGE expression on CECs is involved in the pathophysiology of KD.

\section{METHODS}

The study was approved by the ethical committee of the Children's Hospital, Zhejiang University School of Medicine, and based on the institution's guidelines for human studies. The investigation conformed to the principles outlined in the Declaration of Helsinki. Informed consent was obtained from all the subjects.

\section{Subjects}

Eighty-nine KD patients (52 males and 37 females), aged 3-152 mo $(28.2 \pm 24.6 \mathrm{mo})$ were enrolled. The duration of fever was $4-10 \mathrm{~d}$ (5.6 $\pm 3.4 \mathrm{~d}$ ). The erythrocyte sedimentation rate and C-reactive protein were $78 \pm 33 \mathrm{~mm} / \mathrm{h}$ and $92 \pm 55 \mathrm{mg} / \mathrm{l}$, respectively, in acute-stage $\mathrm{KD}$ patients, and $87 \pm 34 \mathrm{~mm} / \mathrm{h}$ and $13 \pm 25 \mathrm{mg} / \mathrm{l}$, respectively, in subacutestage KD patients. All the patients were treated with IVIG at $1 \mathrm{~g} / \mathrm{kg} / \mathrm{d}$ for $2 \mathrm{~d}$ and oral aspirin at $30-50 \mathrm{mg} / \mathrm{kg} / \mathrm{d}$. After $3-5 \mathrm{~d}$ of treatment, when the patient's temperature returned to normal, the dose of aspirin was reduced to $3-5 \mathrm{mg} / \mathrm{kg} / \mathrm{d}$ for $12 \mathrm{wk}$. There were seven IVIGresistant $\mathrm{KD}$ patients ( 5 males and 2 females), aged 6-122 mo (24.8 $\pm 25.4 \mathrm{mo}$ ), whose temperature was still higher than $38^{\circ} \mathrm{C}$ after $48 \mathrm{~h}$ of standard treatment; these were continued with IVIG at $1 \mathrm{~g} / \mathrm{kg} / \mathrm{d}$ for another $2 \mathrm{~d}$. All the KD patients fulfilled the diagnostic criteria established by the Kawasaki Disease Research Committee (31). Fifteen patients ( 9 males and 6 females), aged 6-97 mo $(23.8 \pm 21.2 \mathrm{mo})$, were found with CALs (defined as coronary artery $z$-score $>5$ (32)) by echocardiography during the disease process; four of the patients with CALs had IVIG-resistant KD. Dipyridamole and warfarin were added in patients with CALs depending on the severity of CALs.

The disease process durations between 4 and $10 \mathrm{~d}, 11$ and $21 \mathrm{~d}$, and 22 and $60 \mathrm{~d}$ were defined as A-KD, SA-KD, and C-KD, respectively. Thirty-eight healthy children (21 males and 17 females), aged 5-144 mo $(27.6 \pm 26.2 \mathrm{mo})$, were enrolled in the control group.

\section{Blood Samples}

Two milliliters of venous blood samples were collected in $89 \mathrm{KD}$ patients (three times for each patient), including acute stage before IVIG treatment, subacute stage (mean (range): 15 (13-18) d), and convalescent stage (30 (28-34) d), and in 38 healthy children, and anticoagulated with 1:10 heparin. Anticoagulated blood samples were kept at $4{ }^{\circ} \mathrm{C}$ and analyzed by flow cytometry within $2 \mathrm{~h}$ of venesection. The samples were divided into two tubes (each with $100 \mu$ l): one tube was used for testing by adding $10 \mu \mathrm{l}$ monoclonal goat antihuman RAGE antibody (R\&D systems), and the other was used as control by adding goat IgG1. After $30 \mathrm{~min}$, both tubes were washed once using PBS, then incubated with fluorescein isothiocyanate-labeled rabbit anti-goat IgG (Southern Biotech) for $30 \mathrm{~min}$. After washing twice with $\mathrm{PBS}$ and removing the supernatant, the control tube was stained with $5 \mu \mathrm{l}$ CD45-PerCP mAb (CD45 monoclonal antibody was conjugated with peridinin-chlorophyll- $\alpha$-protein, USA Becton Dickinson) and mouse IgG1-Phycoerythrin (PE), while the testing tube was stained with $5 \mu \mathrm{l} \mathrm{CD} 45$-PerCP $\mathrm{mAb}$ and $5 \mu \mathrm{l} \mathrm{CD146-PE} \mathrm{mAb} \mathrm{(CD146} \mathrm{mono-}$ clonal antibody was conjugated with R-phycoerythrin, USA Becton Dickinson). After incubation at $4^{\circ} \mathrm{C}$ for $20 \mathrm{~min}, 400 \mu \mathrm{l}$ hemolytic agent (FACSTM, USA Becton Dickinson) was added to each of the two tubes; after $10 \mathrm{~min}$ the tubes were centrifuged at 1,000 rpm for $10 \mathrm{~min}$. Finally, $500 \mu \mathrm{l}$ PBS was added to each of the two tubes and mixed well to be detected after removal of the supernatant.

\section{Flow Cytometry}

The samples in both tubes were evaluated by flow cytometry (FACS Calibur, USA Becton Dickinson). Flow cytometry data were analyzed with CellQuest software. A multistep manual technique was used to detect and quantify the CECs and RAGE on surface of the CECs; 100,000 cells were detected including mononuclear cells (lymphocytes, monocytes, and CECs) and polymorphonuclear leukocytes, and data were kept in the computer. Excitation light sources were $488 \mathrm{~nm}$ for fluorescein isothiocyanate, $\mathrm{PE}$, and PerCP.
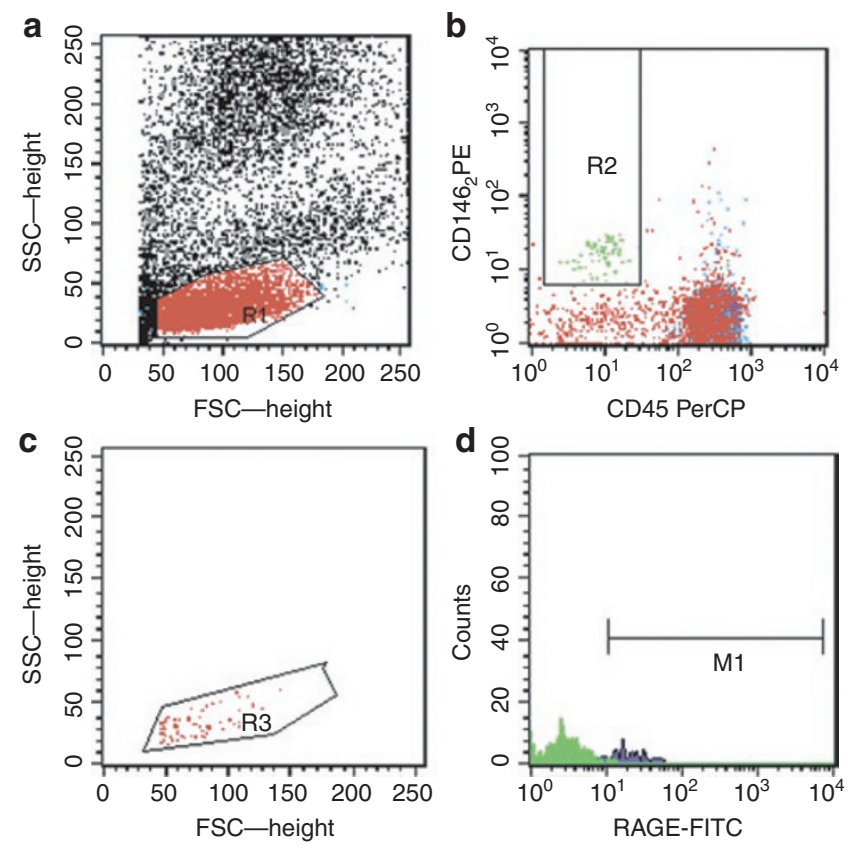

Figure 3. Diagram of CECs-RAGE/CECs and FI-RAGE-CECs measurement by flow cytometry. (a) Screening of mononuclear cells (R1); (b) screening of CECs (R2); (c) verifying the CECs again (R3); (d) measuring CECs-RAGE/ CECs and FI-RAGE-CECs (green represents the isotype control scan). M1: scope of RAGE-positive CECs. CECs, circulating endothelial cells; FI, fluorescence intensity; FITC, fluorescein isothiocyanate; FSC, forward scatter characteristics; RAGE, receptor for advanced glycation end products; PerCP, peridinin-chlorophyll-a-protein; SSC, side scatter characteristics. 


\section{Gating and Quantification of CECs and RAGE on the Surface of CECs}

The first step was to screen mononuclear cells (R1) (Figure 3a), and the second step was to screen CECs (Figure 3b). Monocytes and lymphocytes were excluded by CD45 staining, and CECs were gated by low forward scatter characteristics and low side scatter characteristics. CECs were identified if the cells were $\mathrm{CD} 146^{+} \mathrm{CD} 45^{-}$cells (33). A region (R2) of CD146 ${ }^{+} \mathrm{CD} 45^{-}$cells with known light scatter properties was labeled CECs. The third step was to verify the CECs again (R3) (Figure 3c) and to calculate the ratio of CECs to mononuclear cells (CECs/MNC). The last step was to measure the positive rate of RAGE on the surface of CECs (CECs-RAGE/CECs) and the FI of RAGE on the surface of CECs (FI-RAGE-CECs) (Figure 3d).

\section{Statistical Analysis}

Analysis of the difference between the groups of patients was accomplished using ANOVA followed by least significant difference multiple comparison for normal data and Mann-Whitney test for nonparametric data. The difference was considered significant with a $P$ value $<0.05$. The results were expressed as mean \pm SD. All analysis was implemented in SPSS 16.0 Software.

\section{STATEMENT OF FINANCIAL SUPPORT}

This work was supported by the National Nature Scientific Research Foundation (30872799) and the Zhejiang Nature Scientific Research Foundation (Y207201) in China.

\section{REFERENCES}

1. Cuttica RJ. Vasculitis, Kawasaki disease, and pseudovasculitis. Curr Opin Rheumatol 1997;9:448-57.

2. Rowley AH, Shulman ST. Kawasaki syndrome. Clin Microbiol Rev 1998;11:405-14.

3. Taubert KA, Rowley AH, Shulman ST. Nationwide survey of Kawasaki disease and acute rheumatic fever. J Pediatr 1991;119:279-82.

4. Suzuki A, Kamiya T, Arakaki Y, Kinoshita Y, Kimura K. Fate of coronary arterial aneurysms in Kawasaki disease. Am J Cardiol 1994;74:822-4.

5. Fujiwara T, Fujiwara H, Nakano H. Pathological features of coronary arteries in children with Kawasaki disease in which coronary arterial aneurysm was absent at autopsy. Quantitative analysis. Circulation 1988;78:345-50.

6. Fulton DR, Newburger JW. Long-term cardiac sequelae of Kawasaki disease. Curr Rheumatol Rep 2000;2:324-9.

7. Kato H, Sugimura T, Akagi T, et al. Long-term consequences of Kawasaki disease. A 10- to 21-year follow-up study of 594 patients. Circulation 1996;94:1379-85.

8. Leung DY, Schlievert PM, Meissner HC. The immunopathogenesis and management of Kawasaki syndrome. Arthritis Rheum 1998;41:1538-47.

9. Nakamura Y, Yanagawa H, Harada K, Kato H, Kawasaki T. Mortality among persons with a history of Kawasaki disease in Japan: existence of cardiac sequelae elevated the mortality. J Epidemiol 2000;10:372-5.

10. Newburger JW, Takahashi M, Gerber MA, et al.; Committee on Rheumatic Fever, Endocarditis and Kawasaki Disease; Council on Cardiovascular Disease in the Young; American Heart Association; American Academy of Pediatrics. Diagnosis, treatment, and long-term management of Kawasaki disease: a statement for health professionals from the Committee on Rheumatic Fever, Endocarditis and Kawasaki Disease, Council on Cardiovascular Disease in the Young, American Heart Association. Circulation 2004;110:2747-71.

11. Koc M, Richards HB, Bihorac A, Ross EA, Schold JD, Segal MS. Circulating endothelial cells are associated with future vascular events in hemodialysis patients. Kidney Int 2005;67:1078-83.
12. Nakatani K, Takeshita S, Tsujimoto H, Kawamura Y, Tokutomi T, Sekine I. Circulating endothelial cells in Kawasaki disease. Clin Exp Immunol 2003; 131:536-40.

13. Miranda LP, Tao T, Jones A, et al. Total chemical synthesis and chemotactic activity of human S100A12 (EN-RAGE). FEBS Lett 2001;488:85-90.

14. Foell D, Roth J. Proinflammatory S100 proteins in arthritis and autoimmune disease. Arthritis Rheum 2004;50:3762-71.

15. Foell D, Wittkowski H, Vogl T, Roth J. S100 proteins expressed in phagocytes: a novel group of damage-associated molecular pattern molecules. J Leukoc Biol 2007;81:28-37.

16. Moroz OV, Antson AA, Dodson EJ, et al. The structure of S100A12 in a hexameric form and its proposed role in receptor signalling. Acta Crystallogr D Biol Crystallogr 2002;58(Pt 3):407-13.

17. Roth J, Vogl T, Sorg C, Sunderkötter C. Phagocyte-specific S100 proteins: a novel group of proinflammatory molecules. Trends Immunol 2003;24:155-8.

18. Rouleau P, Vandal K, Ryckman C, et al. The calcium-binding protein S100A12 induces neutrophil adhesion, migration, and release from bone marrow in mouse at concentrations similar to those found in human inflammatory arthritis. Clin Immunol 2003;107:46-54.

19. Moroz OV, Dodson GG, Wilson KS, Lukanidin E, Bronstein IB. Multiple structural states of S100A12: A key to its functional diversity. Microsc Res Tech 2003;60:581-92.

20. Schmidt AM, Yan SD, Yan SF, Stern DM. The multiligand receptor RAGE as a progression factor amplifying immune and inflammatory responses. J Clin Invest 2001;108:949-55.

21. Hofmann MA, Drury S, Fu C, et al. RAGE mediates a novel proinflammatory axis: a central cell surface receptor for S100/calgranulin polypeptides. Cell 1999;97:889-901.

22. Xie J, Burz DS, He W, Bronstein IB, Lednev I, Shekhtman A. Hexameric calgranulin C (S100A12) binds to the receptor for advanced glycated end products (RAGE) using symmetric hydrophobic target-binding patches. J Biol Chem 2007;282:4218-31.

23. Burns JC. S100 proteins in the pathogenesis of Kawasaki disease. J Am Coll Cardiol 2006;48:1265-7.

24. Ebihara T, Endo R, Kikuta H, et al. Differential gene expression of S100 protein family in leukocytes from patients with Kawasaki disease. Eur J Pediatr 2005;164:427-31.

25. Foell D, Ichida F, Vogl T, et al. S100A12 (EN-RAGE) in monitoring Kawasaki disease. Lancet 2003;361:1270-2.

26. Ye F, Foell D, Hirono KI, et al. Neutrophil-derived S100A12 is profoundly upregulated in the early stage of acute Kawasaki disease. Am J Cardiol 2004;94:840-4.

27. Fu S, Gong F, Xie C, et al. S100A12 on circulating endothelial cells surface in children with Kawasaki disease. Pediatr Res 2010;68:165-8.

28. Taguchi A, Blood DC, del Toro G, et al. Blockade of amphoterin/ RAGE signalling suppresses tumor growth and metastases. Nat Lond 2000;405:354-60.

29. Mahajan N, Bahl A, Dhawan V. C-reactive protein (CRP) up-regulates expression of receptor for advanced glycation end products (RAGE) and its inflammatory ligand EN-RAGE in THP-1 cells: inhibitory effects of atorvastatin. Int J Cardiol 2010;142:273-8.

30. Zhong Y, Li SH, Liu SM, et al. C-Reactive protein upregulates receptor for advanced glycation end products expression in human endothelial cells. Hypertension 2006;48:504-11.

31. Ayusawa M, Sonobe T, Uemura S, et al.; Kawasaki Disease Research Committee. Revision of diagnostic guidelines for Kawasaki disease (the 5th revised edition). Pediatr Int 2005;47:232-4.

32. Manlhiot C, Millar K, Golding F, McCrindle BW. Improved classification of coronary artery abnormalities based only on coronary artery z-scores after Kawasaki disease. Pediatr Cardiol 2010;31:242-9.

33. Khan SS, Solomon MA, McCoy JP Jr. Detection of circulating endothelial cells and endothelial progenitor cells by flow cytometry. Cytometry B Clin Cytom 2005;64:1-8. 